\title{
Tak-Tak-Tak, una lectura desde la propuesta de innovación de Fe y Alegría Colombia
}

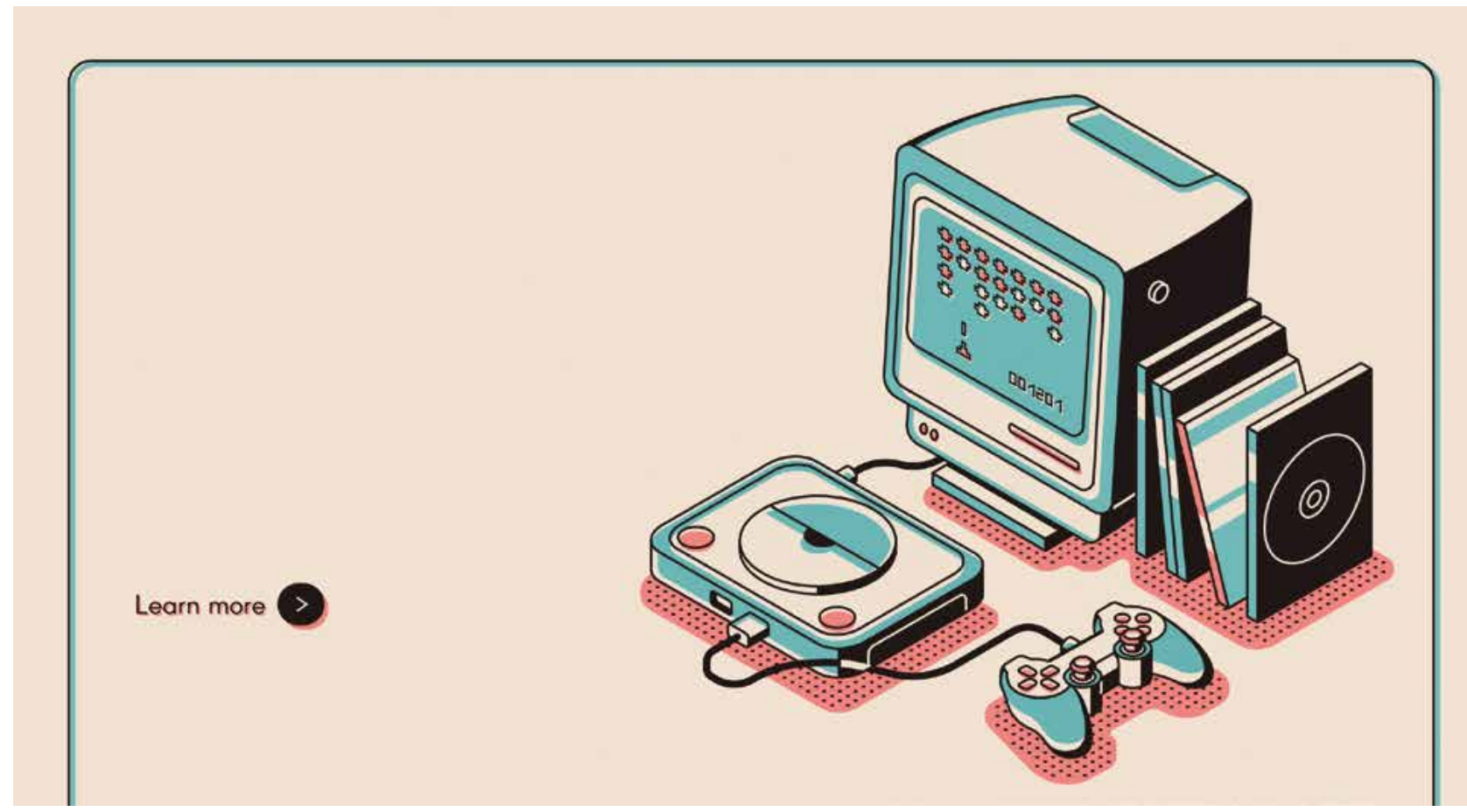

Desde el enfoque de ampliación de capacidades y competencias que suscribe Fe y Alegría Colombia, se está realizando una propuesta educativa innovadora para el aprendizaje y la trasformación social. Con el fin de dar respuesta a Bravo Hernández las necesidades educativas de estos tiempos, que reclaman la integración de software educativo y otras herramientas de corte digital y tecnológico, se ha incluido la iniciativa de la Federación Internacional institucional de Tak-Tak-Tak en esta propuesta para contribuir al fortalecimiento de los aprendizajes del estudiantado. Claudia Marcela Vega González

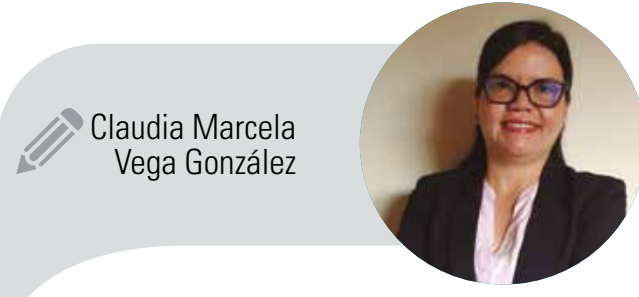

Fe y Alegría Colombia pedagogia.marcela@feyalegria.org.co www.feyalegria.org.co (y) @FeyAlegriaCOL 


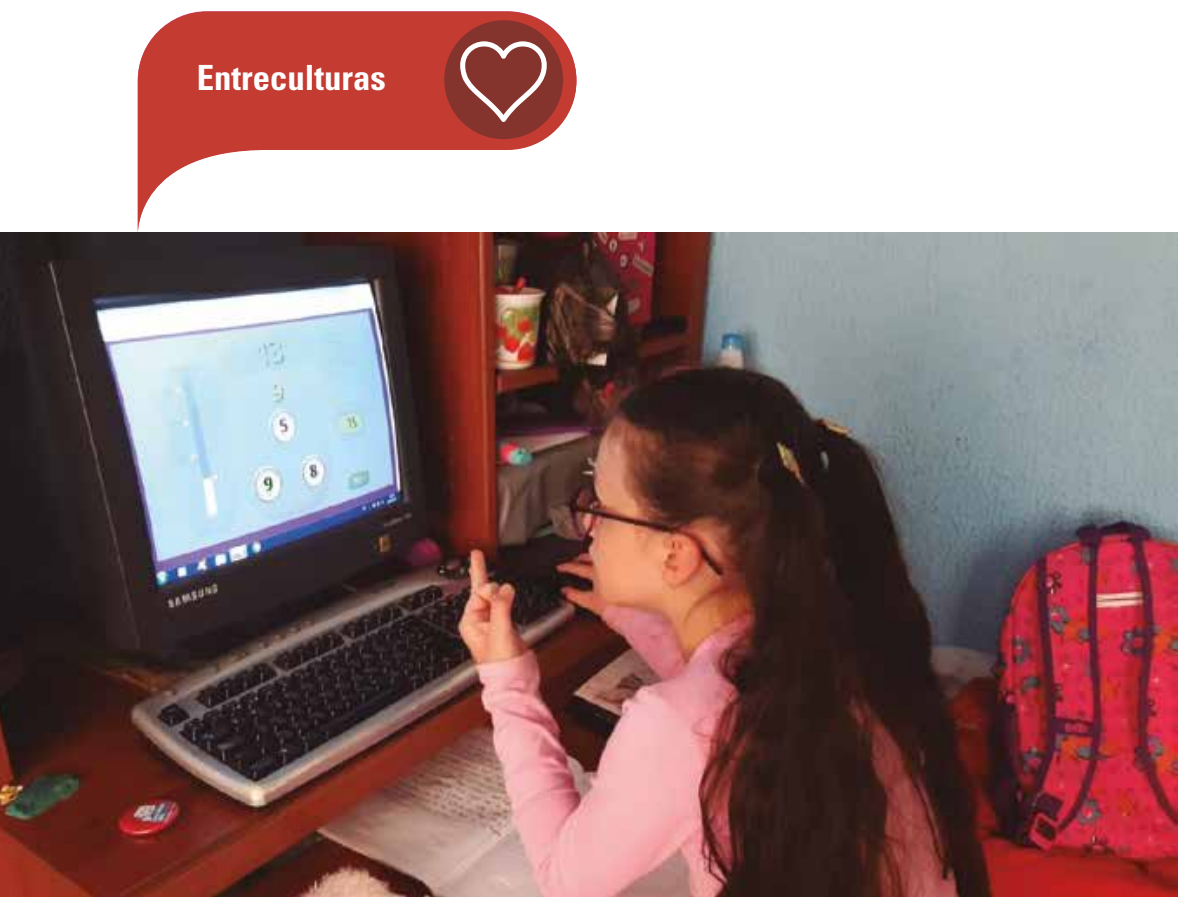

blandas, en la terminología en boga) que se trabaja en el campo educativo. Desde esta perspectiva se entiende la acción en nuestros centros como una preocupación por el desarrollo de las condiciones académicas, pero también sociales, políticas, educativas, culturales que hagan posible esta constitución de una vida humana plena.

Mejía (2020, p. 242) plantea que la opción de convertir las competencias STEM (acrónimo de Science, Technology, Engineering, Mathematics, más la lectura y es-

\section{El enfoque de capacidades en la propuesta educativa de Fe y Alegría}

La propuesta de Innovación para el aprendizaje y la transformación social de Fe y Alegría Colombia (FyACol) incorpora en sus fundamentos el enfoque de capacidades humanas de Martha Nussbaum, señalando "dos aspectos importantes de resaltar: uno, aquello que es capaz de ser y hacer la persona o el grupo social para vivir de manera digna, plena y satisfactoria atendiendo a sus aspiraciones; y el otro, el poder elegir la manera en que realizarán esa vida y tener garantizadas las posibilidades para hacerlo. Cuando no están dadas estas condiciones se deben llevar a cabo las acciones ciudadanas necesarias para exigirlas" (FyACol, 2020, p. 12).

La formación de y en humanidad pasa por la potenciación de las capacidades, entendidas no solo como la carga genética individual, sino como todas las condiciones históricas y sociales que permiten ese florecimiento. De acuerdo con Mejía (2020, p. 129), Gadamer leyendo a Herder afirma que "el término formación designa, de manera fundamental, el modo específicamente humano de dar forma a las disposiciones y capacidades del hombre, que el proceso de formación de una persona lleva implícito el desarrollo de sus potencialidades". La educación, entendida como formación integral, permite ese desarrollo de las capacidades y su desarrollo como habilidades, que al aplicarlas con un alto grado de experticia (y excelencia) se pueden comparar y exhibir como desempeños "competentes". Esta es una primera precisión respecto del difundido concepto de competencias (duras y critura para ellos) como los ejes rectores de la educación de estos tiempos revela que el capitalismo actual está interesado en formar centrándose mucho en el saber hacer, con lo cual se renuncia al carácter integral de la educación.

De otro lado, Nussbaum (2012, pp. 143-145) señala una lista de capacidades humanas: vida; salud; integridad corporal; sentidos, imaginación y pensamiento; emociones; razón práctica; afiliación; otras especies; juego; y control del propio entorno, pero nos advierte que:

Si tenemos la intención de formar adultos que tengan todas las capacidades de la lista, esto significará [...] exigir ciertos tipos de funcionamiento en los niños, [...] a menudo es preciso ejercitar una función en la infancia para producir una capacidad madura en la adultez. Así, parece perfectamente legítimo exigir la educación primaria y secundaria, dado el papel que la misma desempeña en todas las opciones posteriores de una vida adulta. En forma semejante, parece legítimo insistir en la salud, en el bienestar emocional, en la integridad corporal y en la dignidad de los niños. Algo de esta insistencia la formularán los padres, pero el Estado tiene el legítimo papel de impedir el abuso y la negligencia.

(Nussbaum, 2012, p. 160)

A veces, el papel del Estado en la producción de adultos que tengan todas las capacidades de la lista se comprende de manera estrecha, concentrándose en la alfabetización y en otras habilidades básicas, importantes para el desarrollo técnico y económico, y tal vez también en habilidades políticas entendidas en sentido estricto. Mi argumento se opone enfáticamente a un enfoque tan estrecho. A fin de hacer lo que deberían para sus ciudadanos, los Estados deben ocuparse de todas las capacidades, aun cuando estas no parezcan tan útiles para el crecimiento económico 


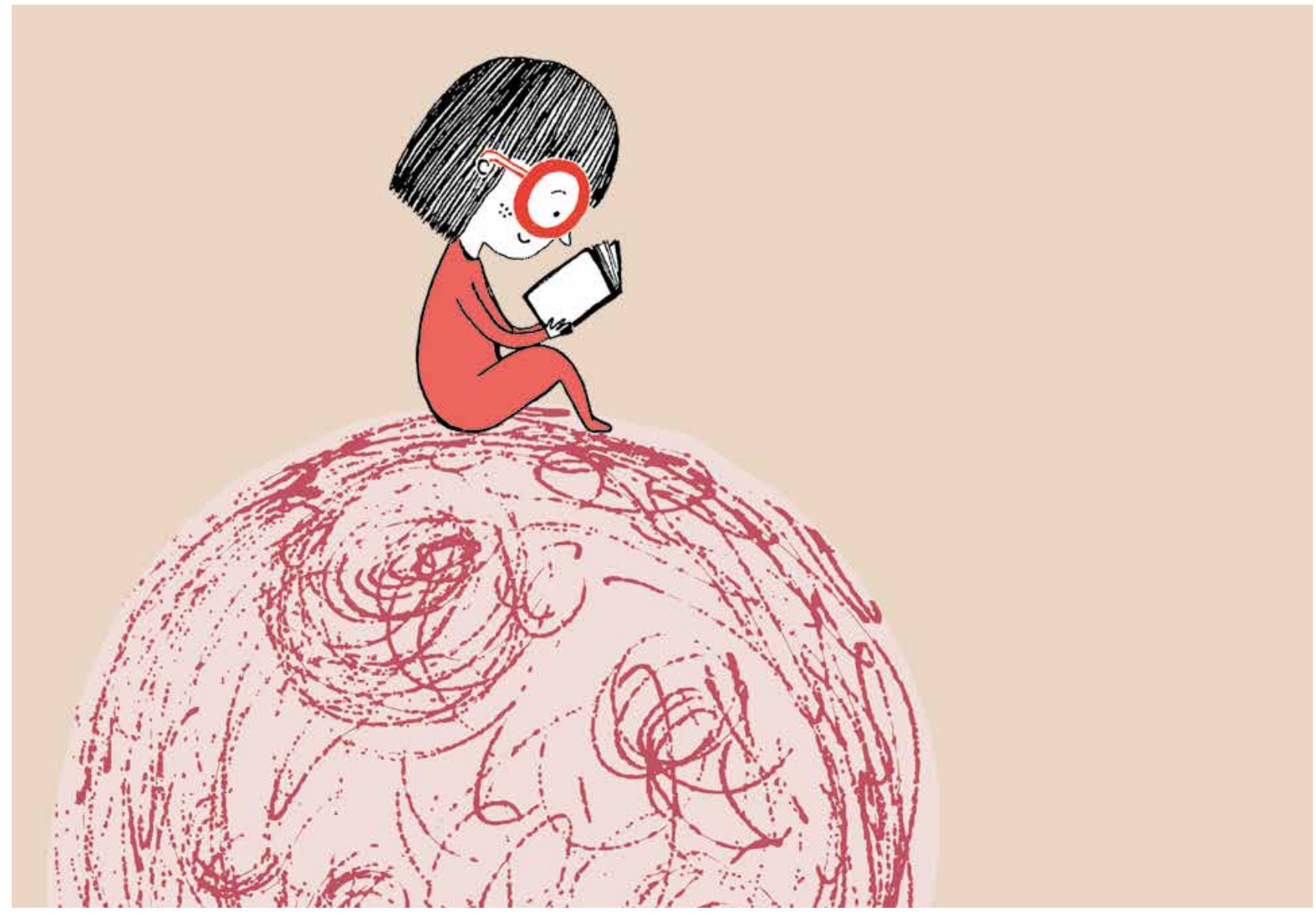

o incluso para el funcionamiento político. Un caso de particular interés es el de la capacidad para lo lúdico.

(Nussbaum, 2012, p. 161)

Hablar de capacidades desde la educación popular significa preguntarse cómo se constituye y se mantiene social e históricamente la integralidad de lo humano y su dignidad, planteando una opción de transformación y emancipación. Es desde allí que la propuesta de innovación de Fe y Alegría Colombia esboza sus cuatro ambientes de aprendizaje (FyACol, 2019, p. 14): Proyectos Interdisciplinares con Incidencia Comunitaria (PIIC), Potenciando Talentos e Intereses (PTI), Cualificación de habilidades, Conceptos y Saberes (Cualificar) y Ciudadanías para la Convivencia la Reconciliación y la Paz (CCRP). Estos ambientes integran todas las áreas básicas y obligatorias establecidas por la legislación educativa colombiana: ciencias sociales, historia, geografía, constitución política y democracia; educación ética y en valores humanos; educación religiosa; humanidades, lengua castellana e idiomas extranjeros; filosofía; ciencias económicas y políticas; ciencias naturales y educación

\section{El capilalismo actual está \\ interesado en formar \\ centrándose mucho en el saber hacer, con lo cual se renuncia al carácler integral de la educación}

ambiental; tecnología e informática; matemáticas; educación física, recreación y deporte; educación artística. En los ambientes se fomenta el autoaprendizaje, el trabajo colaborativo y el desarrollo del pensamiento crítico y creativo.

Por ello, cuando se quiere integrar las TIC (tecnologías de la información y la comunicación), esto es, todos aquellos recursos, herramientas y programas utilizados en el procesamiento, la administración y el intercambio de información mediante diversos soportes tecnológicos a un tipo de educación integral y desde un enfoque de capacidades como el que hemos descrito en los párrafos ante- 


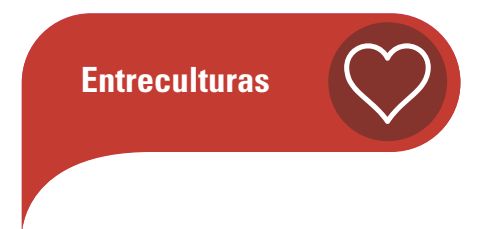

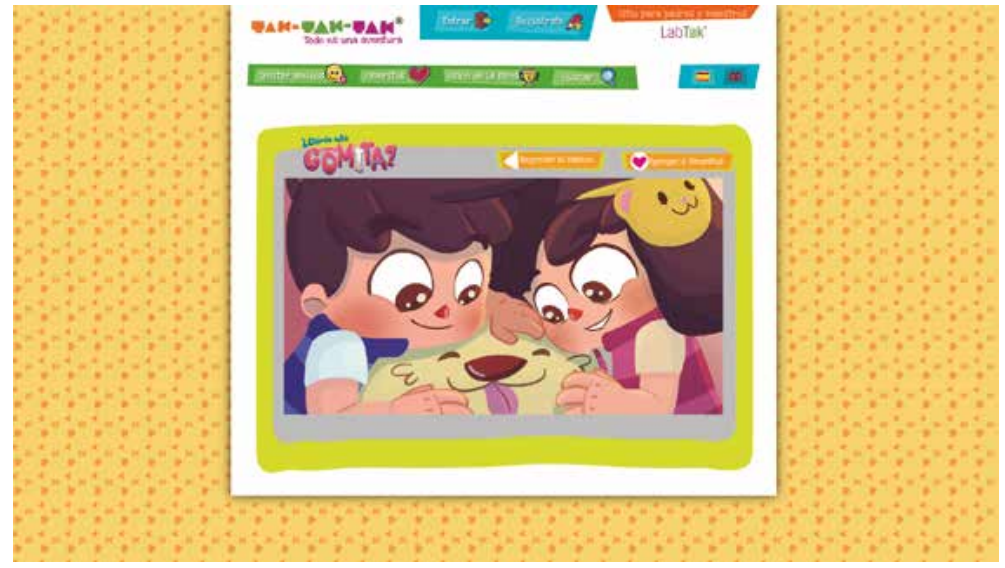

La introducción de las TIC en

la vida colidiana es capaz

de cambiar no solo nuestras

maneras de actuar sino

también las de pensar

riores, nos hacemos algunas preguntas sobre su posible impacto en ella. Como lo enuncia Mejía (2020, p. 15), las TIC en las aulas plantean "dinámicas que van de la simple modernización de la pedagogía, pasando por posiciones que la reducen a un instrumentalizador de técnicas y uso de las tecnologías que, a manera de una ferretería moderna, la trabaja sin lógica, sin lenguajes, sin nuevas realidades, sin algoritmos". Pero lo que sí se sabe que sucede es que la introducción de las TIC en la vida cotidiana es capaz de cambiar no solo nuestras maneras de actuar sino también las de pensar.

\section{El lugar de Tak-Tak-Tak en la propuesta educativa}

En este sentido, para la innovación en marcha, la experiencia con los videojuegos educativos de Tak-Tak-Tak es una primera aproximación a su integración en el trabajo de aula de una manera extendida. El proyectoTak-Tak-Tak fue creado por Inoma AC, organización mexicana no gubernamental y sin fines de lucro fundada en 2011. En idioma NáhuatI, Inoma quiere decir "por ti mismo". Tak-tak-tak es un sis- tema gratuito de videojuegos educativos que ofrece a los usuarios una experiencia individualizada con opciones de juego y aprendizaje acordes a sus conocimientos y capacidades. El sistema tiene como objetivo apoyar, complementar y reforzar la educación en un entorno atractivo y entretenido, principalmente para niños y niñas de 6 a 12 años. Busca generar el dominio de la tecnología, la solución de problemas y el trabajo en equipo, así como impulsar aprendizajes específicos de diversas asignaturas (Inoma, 2021, p. 1).

Es importante referir que estos videojuegos se construyeron con base en mapas curriculares de secuencias de aprendizajes que aumentan en grado de dificultad, diversidad de conocimientos y experiencias con el propósito de guiar el desarrollo de conceptos dentro de una misma rama de conocimiento (Inoma, 2021, p. 1). De esta manera, las niñas y los niños son retados a avanzar en el juego al tiempo que consolidan conceptos.

Inoma (2020) en su propuesta, al igual que la de Fe y Alegría Colombia, trabaja por la transformación de la práctica docente, el logro de los aprendizajes y la mejora de la calidad educativa, aspectos fundamentales para consolidar una acción educativa innovadora.

Con respecto a los principios de la propuesta de Tak-Tak-Tak, podemos referir los siguientes:

$\pi$ El referente fundamental del aprendizaje es el estudiantado. Por ello, los videojuegos plantean el desarrollo de competencias relacionadas con el componente académico como el desarrollo de habilidades superiores del pensamiento para solucionar problemas, pensar críticamente, comprender y explicar situaciones desde diversas áreas del saber, manejar información e innovar. Lo anterior se complementa con juegos que trabajan componentes emocionales y valores como la responsabilidad social, cívica y ética.

$\boldsymbol{\lambda}$ El uso de videojuegos genera un ambiente propicio para el aprendizaje al proporcionar experiencias diferentes 


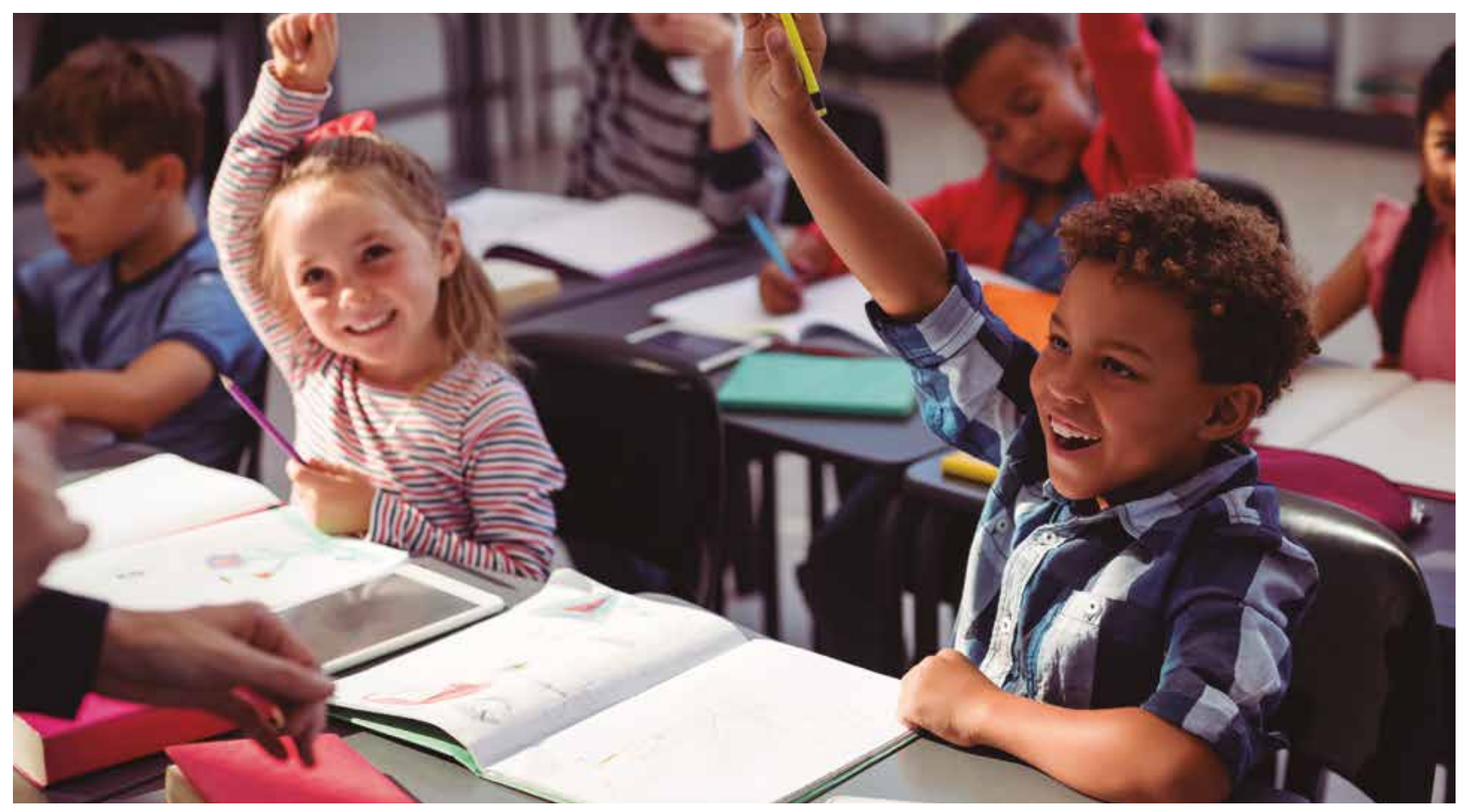

a las ofrecidas en el aula presencial y que van de la mano de las nuevas tecnologías.

7 A través de la retroalimentación de los juegos, las y los estudiantes se sienten motivados a continuar, lo que ayuda a su autoestima.

En la experiencia, hasta donde vamos, un grupo de nuestras docentes han identificado como aciertos en el uso del material, los siguientes:

1. La mediación del profesorado es fundamental para el logro de los aprendizajes que se plantean desde los videojuegos. De allí la importancia de que las y los docentes conozcan e identifiquen con claridad los alcances y los conceptos que potencia cada uno, de tal manera que puedan ubicarlos en sus planificaciones.

2. El uso de la plataforma fortalece tanto el componente académico como el socioemocional. A nivel conceptual favorece el desarrollo de habilidades numéricas, de patrones, representación, comprensión, seguimiento de instrucciones, entre otras. En el campo socioemocional, promueven el manejo de los sentimientos, la perseverancia y la cooperación.

3. Facilitan el trabajo en equipo y las acciones colaborativas, aspectos que se evidencian en los momentos de explicación y cuando las niñas y los niños socializan maneras de superar los retos del juego.

4. Potencian en el estudiantado la lectura y comprensión de instrucciones.

5. Favorecen la planificación al momento de diseñar las estrategias del juego.

6. El componente lúdico conecta y motiva al estudiantado para aprender.

7. Las herramientas de seguimiento que maneja el profesorado, permiten identificar el nivel de avance de cada estudiante con respecto al juego y los conceptos que este desarrolla.

8. El equipo docente ha motivado a las familias a acompañar a las niñas y los niños cuando usan la herramienta, lo que ha facilitado su involucramiento.

9. Potencian la autonomía y el cumplimiento del logro en perspectiva de lo que referimos en nuestra propuesta educativa como excelencia.

En la actualidad llevamos 10 meses de implementación y se encuentra en marcha una evaluación a nivel federativo, que busca encontrar la "Influencia de los videojuegos en la competencia matemática de niños y niñas de colegios de la Federación Fe y Alegría".

Para fines del presente artículo recogimos algunos testimonios significativos de maestras que vienen empleando los 

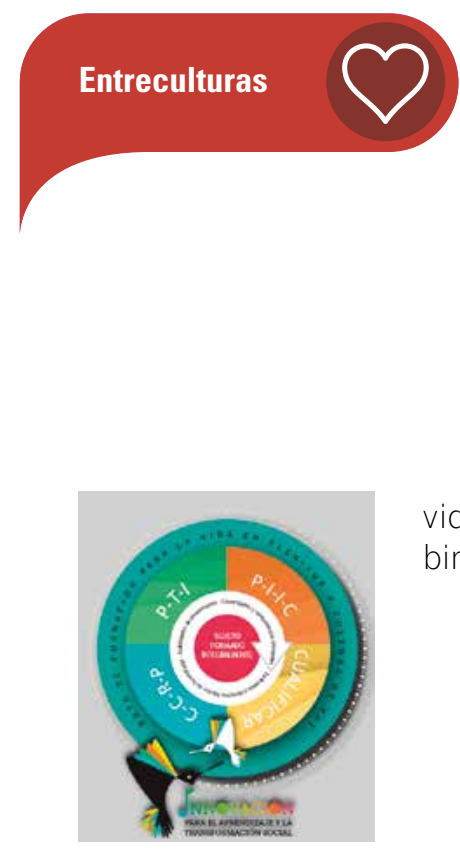

videojuegos en sus aulas y aquí transcribimos algunos apartes:

“Esta plataforma en el trabajo académico de nuestros estudiantes ha sido muy importante y de gran utilidad, ya que posee características intrínsecas que ayudan a los niños y niñas a desarrollar las habilidades cognitivas, socioemocionales y motrices apoyando el desarrollo de las competencias planteadas desde la lógico-matemática y la comprensión lectora, porque les exige leer y comprender instrucciones. También, ha permitido avanzar en el trabajo colaborativo, ya que cuando nos reunimos e interactuamos como grupo, socializamos la manera de superar retos en el juego.

[...] Las docentes hemos explorado y encontrando juegos que se ajustan a los diferentes ambientes de aprendizaje, relacionados no solo con Cualificar, sino también con los temas PIIC. Los juegos más utilizados por los estudiantes de transición a tercero son: Caracol, Mundial Animal, Boris, Cañón Tortuga, Tilín-Tilín, ¿Dónde está gomita? o Cucarachilandia, entre otros". Nubia Chacón. Docente Las Mercedes IED. Primer ciclo.

“He adaptado los juegos de la plataforma a cada uno de los ambientes de aprendizaje. En lo que he venido trabajando este año, llevo alrededor de 12 juegos, los trabajo por semana. En base a las indicaciones que aparecen en la plataforma, hacemos las tareas que registramos en una agenda que el grupo acordó llevar [...] por ejemplo: en Maestro Dragón, la tarea es mirar el valor de la perseverancia y ver cómo en la vida real soy perseverante y en qué momento he sido perseverante.

\section{DARA SABER MÂS}

Fe y Alegría Colombia. (2019). Innovación para el aprendizaje y la transformación social. Bogotá: AECID - Fe y Alegría CoIombia.

INOMA. (2021). Fundamento educativo de los videojuegos de TAK-TAK-TAK. Documento interno.

Nussbaum, M. (2012). Las mujeres y el desarrollo humano. El enfoque de las capacidades (Roberto Bernet, trad.). Barcelona: Herder.
[...] También creé un canal de Tak-Tak-Tak en el equipo de Teams para que los niños suban sus tareas y compartan inquietudes.

[...] Lo que me ha gustado de esta plataforma es que los niños la conocen muy bien y con el tiempo hemos aprendido a terminar el juego para que lleguen a las metas y traten de subir niveles o de superar obstáculos".

Diana Otalora. Colegio Torquigua IED. Primer ciclo

En estos testimonios podemos identificar que las maestras complementan su mediación incluyendo en los desarrollos de sus clases un gran número de videojuegos, pero ellas mantienen la dirección del proceso pedagógico. Asimismo, señalan que promueven el trabajo colaborativo entre estudiantes para resolver algunos problemas planteados e incluso se involucra a algunas familias. Estas prácticas muestran algunas modificaciones en los hábitos de estudio de niños y niñas, y se reportan avances en sus aprendizajes, pero tendremos más datos para análisis cuando termine la investigación que se está llevando a cabo.

Como tarea complementaria a dicho proceso estarán las sistematizaciones, que arrojarán información sobre los posibles cambios y sus proyecciones. Somos una experiencia viva y estamos aprendiendo a integrar estas herramientas tecnológicas para la educación, sin olvidar el marco educativo en el que están ubicadas: la realización y ampliación de las capacidades humanas del estudiantado. Ese es nuestro desafío y esperamos cumplirlo de una mejor manera apoyándonos en este nuevo recurso •

\section{HEMOS HABLADO DE}

\section{Capacidades; videojuegos; educación popular; aprendizaje; innovación educativa.}

Este artículo fue solicitado por PADRES Y MAESTROS en marzo de 2021, revisado y aceptado en mayo de 2021. 